\title{
Hard gamma emission close to the GDR energy region
}

\author{
Emil Běták* \\ Institute of Physics, Slovak Academy of Sciences, 84511 Bratislava, Slovakia \\ Faculty of Philosophy and Sciences, Silesian University, 74601 Opava, Czech Republic \\ E-mail: betak@savba.sk
}

\section{Andrej Likar}

Faculty of Mathematics and Physics, Ljubljana University, 1000 Ljubljana, Slovenia

J. Stefan Institute, 1001 Ljubljana, Slovenia

E-mail: andrej.likar@ijs.si

\section{Tim Vidmar}

J. Stefan Institute, 1001 Ljubljana, Slovenia

E-mail: tim.vidmar@ijs.si

\begin{abstract}
We address mainly the nucleon radiative capture reactions at incident energies above $10 \mathrm{MeV}$, but sufficiently low so that bremsstrahlung and the quasi-deuteron mechanisms do not play significant role. The main process considered is the direct-semi-direct capture treated either conventionally as the DSD model or interpreted within statistical approach as the pre-equilibrium $\gamma$ emission. We compare the model assumptions of both approaches and also their applicability and predictions.
\end{abstract}

Workshop on Photon Strength Functions and Related Topics

June 17-20, 2007

Prague, Czech Republic

${ }^{*}$ Speaker. 


\section{Introduction}

Reactions of radiative capture of nucleons - even though very difficult to be measured due to their low cross sections - serve as a challenge for different $\gamma$ emission mechanisms already for decades. There are two competing and complementary models to describe the $\gamma$ emission in the continuum region, namely the direct-semi-direct model (DSD) and the pre-equilibrium decay.

The first one (see [1, 2, 3]), has proved its excellent capabilities in describing the transitions going directly to discrete states with well specified quantum numbers. (i.e. those strongly of singleparticle nature, usually very close to the (doubly-)magic nuclei or at least near closed shells). The states are described by the use of the optical model wave functions.

The pre-equilibrium model (see, e.g., [4]), on the other hand, is in its nature a statistical approach to the problem. Anyway, it appeared to be rather successful to describe the $\gamma$ energy spectra in the continuum region in $14 \mathrm{MeV}$ neutron-induced reactions $[5,6,7]$. The study of excitation functions of both neutron- and proton- induced reactions $[8,9,10,11]$ extended the applicability of the model to energies starting from few $\mathrm{MeV}$ to about $30 \mathrm{MeV}$. Significant improvement was the incorporation of spin into the formalism of the pre-equilibrium exciton model [12,13], which enabled also pre-equilibrium calculations leading to discrete states [14] and the comparison to the direct-semi-direct model calculations [3].

Both models have some common features, even though they are strictly complementary in their underlying physics. Whereas the direct-semi-direct model deals with wavefunctions and specific interactions of nucleons, while completely ignoring competing processes, the pre-equilibrium models are of statistical nature and they deeply involve quantities like the level densities etc. The competition - e.g. of the nucleon emission - is naturally contained there. What is common to both models is that they both are capable to reproduce (more-or-less) the data corresponding to hard $\gamma$ emission [15] observed in radiative nucleon capture at energies above few MeV.

The available experimental data are not frequent due to low cross sections and - at the same time - low detector efficiency for these hard $\gamma$ 's.

The reactions of nucleon radiative capture are also a suitable tool for their deep insight into the details of nuclear reaction process itself. The latter can be studied in more detail by reactions leading to discrete and/or semi-discrete states; more frequently those induced by protons are studied (due to the energy resolution).

\section{Pre-Equilibrium Description and Calculations}

We used the computer codes EMPIRE-II v. 2.19 [16] and TALYS [17]. At excitation energies above $10 \mathrm{MeV}$, they both are based on the pre-equilibrium single-particle radiative mechanism [5, 6], which has been elaborated proved to be very successful at the incident energies below about $30 \mathrm{MeV}$ in previous codes (see, e.g. Refs. [18, 19]). On the other hand, it gives also a good and reliable description at energies as low as about $5 \mathrm{MeV}[9,11]$. The $\gamma$ emission rates can be expressed as $[5,6]$

$$
\lambda_{\gamma}\left(n, E, \varepsilon_{\gamma}\right)=\frac{\varepsilon_{\gamma}^{2} \sigma_{\mathrm{GDR}}\left(\varepsilon_{\gamma}\right)}{\pi^{2} \hbar^{3} c^{2}} \frac{\sum_{m=n, n-2} b\left(m, \varepsilon_{\gamma}\right) \omega\left(m, E-\varepsilon_{\gamma}\right)}{\omega(n, E)},
$$


with $b$ 's the corresponding branching ratios (see [5,6]) and $\omega$ 's the corresponding state densities with specified energy and the number of excitons. and the branching ratios are

$$
\begin{aligned}
b\left(n-2, \varepsilon_{\gamma}\right) & =\frac{\omega\left(2, \varepsilon_{\gamma}\right)}{g(n-2)+\omega\left(2, \varepsilon_{\gamma}\right)} \\
b\left(n, \varepsilon_{\gamma}\right) & =\frac{g n}{g n+\omega\left(2, \varepsilon_{\gamma}\right)} .
\end{aligned}
$$

With the inclusion of spin, however, the branching ratios become much more complicated, but fortunately — they factorize [12],

$$
b_{m S}^{n J}=\frac{y_{m}^{n} x_{m S}^{n J}}{y_{m}^{m} x_{m S}^{m J}+y_{m}^{m+2} x_{m S}^{m+2 J}},
$$

where $y$ 's and the energy-dependent parts are identical to those of the spin-independent ones, and in the case of the equidistant-spacing scheme they become

$$
\begin{gathered}
y_{n}^{n}=g n, \\
y_{n}^{n+2}=g^{2} \varepsilon_{\gamma},
\end{gathered}
$$

and $x$ 's arise from the spin couplings (for the details, see [12]).

The photoabsorption cross section $\sigma_{\mathrm{GDR}}$ is usually taken in the form of the giant dipole resonance approximated by the corresponding Lorentzian (or a double-humped Lorentzian in the case of deformed nuclei).

A counterpart to the non-participating states influencing the (effective) nuclear potential can be found also in the pre-equilibrium statistical picture of the single-particle radiative mechanism. The justification of eq. (2.1) stems from the assumption of the $1 p 1 h$ GDR excitation. The model description of a $\gamma$ capture on an even-even target leads to the particle-hole pair creation. However, the $\gamma$ capture as well as its emission is (are) associated with two different processes - the other one leaves the exciton number unchanged and just shifts a particle (or a hole) in energy. This is the origin of the branching ratios in (2.1) and incorporation of not-involved states in the detailed balance was just the difference between the original [5] and the improved [6] formulation of the pre-equilibrium gamma decay. The justification of this inclusion given in [6] was based on the equilibrium limit and different interpretation of the Brink-Axel hypothesis, so that the present argumentation gives an additional support for that.

There are some differences between TALYS and EMPIRE which are important for the preequilibrium stage of the reaction. Apart of using not identical libraries for different parameters and allowing their users for different advanced options, probably the most significant one is that the basic approach is a two-component one (i.e. distinguishing between the neutrons and the protons) in TALYS, whereas one-component formulation with a charge factor is used in EMPIRE ${ }^{1}$.

Previous calculations done using other codes demonstrated sensitivity of the calculations to the details of the level density parameters, where just a slight shift of the level density parameters can change the cross sections by more than one order of magnitude, and also the influence of the temperature-dependent width of the GDR, which is rather surprising at excitation energies

\footnotetext{
${ }^{1}$ More discussion on the differences of these two codes is in Ref. [20].
} 


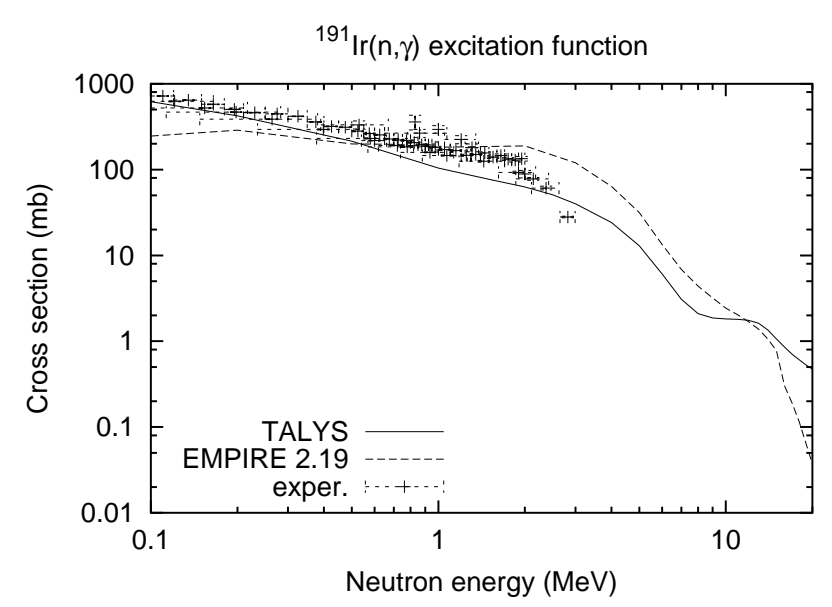

Figure 1: The excitation function of the ${ }^{191} \operatorname{Ir}(\mathrm{n}, \gamma)$ reaction between $100 \mathrm{keV}$ and $20 \mathrm{MeV}$, as calculated by EMPIRE-II v. 2.19 and by TALYS, and compared to the EXFOR experimental data (from Ref. [20]).

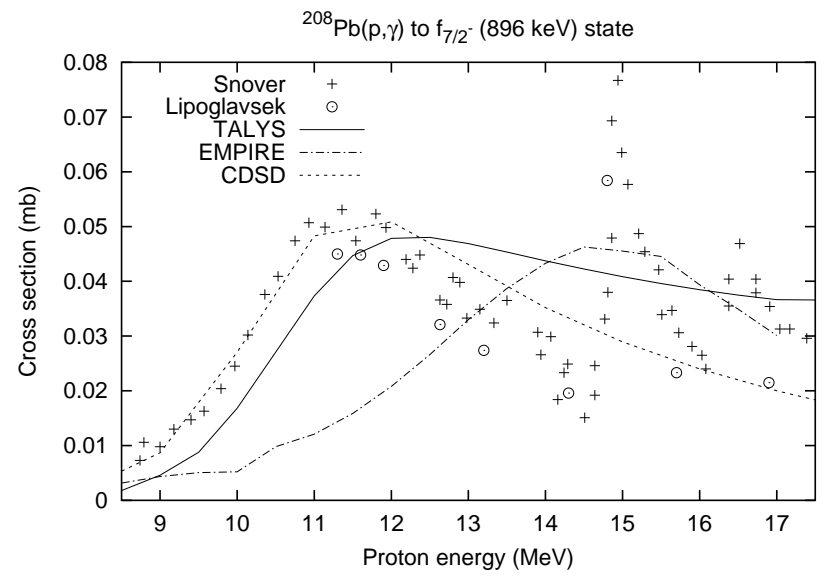

Figure 2: The excitation function of the ${ }^{208} \mathrm{~Pb}(\mathrm{p}, \gamma)$ reaction leading to the $f_{7 / 2^{-}}(896 \mathrm{keV})$ state in ${ }^{209} \mathrm{Bi}$. The experimental data are those of Snover et al. [23] (after their renormalization, see [24]) and of Lipoglavšek et al. [25]. Three sets of calculations are presented, namely the CDSD (Consistent Direct-Semi-Direct model) and those performed by two "do-all" codes including pre-equilibrium $\gamma$ emission, namely EMPIRE-II and TALYS (from Ref. [26]).

well below $50 \mathrm{MeV}$ [9]; the detailed form of the GDR and the differences among different level density models are only of marginal influence and practically may be neglected [21, 22]. Thus, the calculations are relatively reliable far off the closed shells and (simultaneously) close to the betastability line, and rather unsure near closed (or even doubly closed) one(s), where one has to pay the utmost care to the parameters (see, e.g., [9]). Generally, there is no straightforward solution for nuclei close to the drip lines, and calculations performed with different model assumptions and/or codes may serve as a rough estimate how reliable the evaluation may be. For a quality of agreements of the results arising from different computer codes and their fit to the data in the $(\mathrm{n}, \gamma)$ excitation function in wide energy range, see Fig. 1. Fig. 2 indicates, how complicated the comparison may be for discrete states in the vicinity of the doubly-magic nucleus. Therein, also the CDSD calculation [25] is depicted. The sharp fluctuations of experimental data above $E_{p}=14$ $\mathrm{MeV}$ are ascribed to the analog states, which are not considered in the calculations. 


\section{Direct-Semi-Direct Model}

DSD is formally a microscopic approach and wave functions of nuclei (i.e. those strongly of single-particle nature, usually very close to the (doubly-)magic nuclei or at least near closed shells) and of the incident nucleon with well defined quantum numbers are necessary to be known. The interaction is described by the use of the optical model. In doing that, one usually ignores coupling of discrete states involved in the reactions to those in continuum. The price for this neglect is the appearance of non-Hermitian effective Hamiltonians, as recently pointed out by Likar and Vidmar [27].

Ref. [27] has shown — though only within a toy model — that the imaginary part of the (effective) optical model potential arises from the neglect of the coupling of the "participant" state to other(s) (continuum) excited state(s). Similar coupling has been identified several decades ago in atomic physics by Fano [28]. Below, we shall shortly summarize the basic ideas of Ref. [27].

If we consider the ground state $|0\rangle$ and the excited states $\left|\psi_{i}\right\rangle$, which are the eigen states of the Hamiltonian, the total wave function can be written

$$
|\Psi\rangle=\alpha(x)|0\rangle+\sum_{i} \beta_{i}(x)\left|\psi_{i}\right\rangle
$$

where $\alpha$ and $\beta_{i}$ 's are the particle parts of the wave functions, and $x$ stands for all their quantum numbers including energy ${ }^{2}$. Let $V_{i}$ be the interaction part of the Hamiltonian, which couples the ground state to the $i$-th excited one, $\left\langle\psi_{i}|H| 0\right\rangle$. The particle parts $\alpha$ and $\beta_{i}$ 's are coupled together

$$
\begin{array}{r}
\alpha^{2}(x)=\left[1+\sum_{i} \frac{\left|V_{i}\right|^{2}}{E_{i}-E}\right]^{-1}, \\
\beta_{i}(x)=-\frac{V_{i}}{E_{0}-E} \alpha(x) .
\end{array}
$$

As we are interested in the processes involving the ground state, one can eliminate $\beta$ 's from the above equations, what leads to

$$
\left(E-E_{0}\right) \alpha=\sum_{i} \frac{\alpha\left|V_{i}\right|^{2}}{E-E_{i}}
$$

and after introducing the energy in the complex plane, substituting $E$ by $E^{+}=E+i \eta$ and replacing the summation by integration, one arrives to the Breit-Wigner formula with energy $E=E_{0}-i \Gamma / 2$, where the width is $\Gamma=\frac{2 \pi}{D}|V(E)|^{2}$. As a consequence of ignoring the continuum, the effective potential becomes complex here.

If we keep all the states in considerations, we can equally arrive to the Breit-Wigner formula, but without the need to use the complex potential. Thus, the imaginary part is the price for our neglect of the other states.

The basic idea was illustrated on a case study of a system (nucleus) having just two states, the ground state and the first excited one, denoted for simplicity $|0\rangle$ and $|1\rangle$, respectively [27]. Further on, let us assume that $V$ is just a zero-range interaction

$$
V=\mathscr{V}(x)\left(a^{\dagger}+a\right)=g \delta(x)\left(a^{\dagger}+a\right),
$$

\footnotetext{
${ }^{2}$ We shall omit writing $x$ in the formulae below in the cases where it does not cause any confusion.
} 
where $a^{\dagger}$ is the operator switching nucleus from $|0\rangle$ to the $|1\rangle$ state and $a$ the opposite one.

Now, let $T$ be the kinetic part of the Hamiltonian (kinetic energy of the particle). Thus,

$$
\left(T+V+H_{2}\right) \Psi=E \Psi
$$

Obviously,

$$
\begin{array}{r}
(T+\varepsilon-E) \beta(x)=-\mathscr{V}(x) \alpha(x) \\
\beta(x)=-g G_{\beta}^{+}(x) \alpha(x)
\end{array}
$$

and

$$
\begin{array}{r}
T \alpha(x)+\frac{g^{2}}{2 k_{\beta}}(-i \delta(x) \alpha(x))=E \alpha(x) \\
k_{\beta}^{2}=E-\varepsilon
\end{array}
$$

and the absorptive potential is

$$
V(x)=-i \frac{g^{2}}{2 k_{\beta}} \delta(x)
$$

Thus, we arrive to the conclusions that

- The wave function of the ground state is not only $\alpha|0\rangle$, but it has its $|1\rangle$ component as well. The full transition amplitude of the radiative nucleon capture from the initial state $|\Psi\rangle$ to the final ground state $\left|\Psi_{g}\right\rangle$ is $T_{\gamma}=\left\langle\Psi_{i}\left|H_{\gamma}\right| \Psi_{g}\right\rangle=\int \alpha^{*} H_{\gamma} \alpha \mathrm{d} x+\int \beta^{*} H_{\gamma} \beta \mathrm{d} x$. Usually, only the first term is taken into account.

- After using the specific zero-range interaction, the transition amplitude is $T_{\gamma}=\int \alpha^{*}\left[H_{\gamma}+g^{2} G_{\beta}^{*(+)} H_{\gamma} G_{g}\right] \alpha_{g} \mathrm{~d} x$, i.e. we get the operator of the electromagnetic transition $H_{\gamma}$ dressed to become an effective operator $\mathscr{H}_{\gamma}=H_{\gamma}\left(1+g^{2} G_{\beta}^{*(+)} G_{g}\right)$.

In the above, the general behaviour is (hopefully) of a general nature; however, the specific formulae have been derived just within the simplified toy model [27].

\section{Gamma spectra in Proton Radiative Capture above $10 \mathrm{MeV}$}

We have applied both approaches, the DSD model and the pre-equilibrium decay, to the reactions of proton radiative capture to discrete states in ${ }^{208} \mathrm{~Pb}(\mathrm{p}, \gamma)$, measured recently by Lipoglavšek et al. [25, 29]. As an example, we give here the $\gamma$ energy spectrum obtained at three energies, namely $14.8,15.7$ and $16.9 \mathrm{MeV}$ in coincidence with the $896 \mathrm{keV} f_{7 / 2^{-}} \rightarrow$ g.s. transition is presented in Fig. $3^{3}$. The overall agreement of the three calculations and the data is reasonable, however, some differences still can be marked (e.g., the peaks corresponding to direct capture to the low-lying discrete states are much more pronounced in the pre-equilibrium calculations than in the DSD ones or in the experimental data).

\footnotetext{
${ }^{3}$ Preliminary calculations of the pre-equilibrium $\gamma$ spectra from this reaction have been published in Ref. [26]. The EMPIRE calculation is given without accounting for $\gamma$ 's after nucleon emission, for better comparison to the DSD calculations, The visible gap in the EMPIRE spectrum near $18 \mathrm{MeV}$ is just an artefact of insufficient dimension of the computer output table listing.
} 

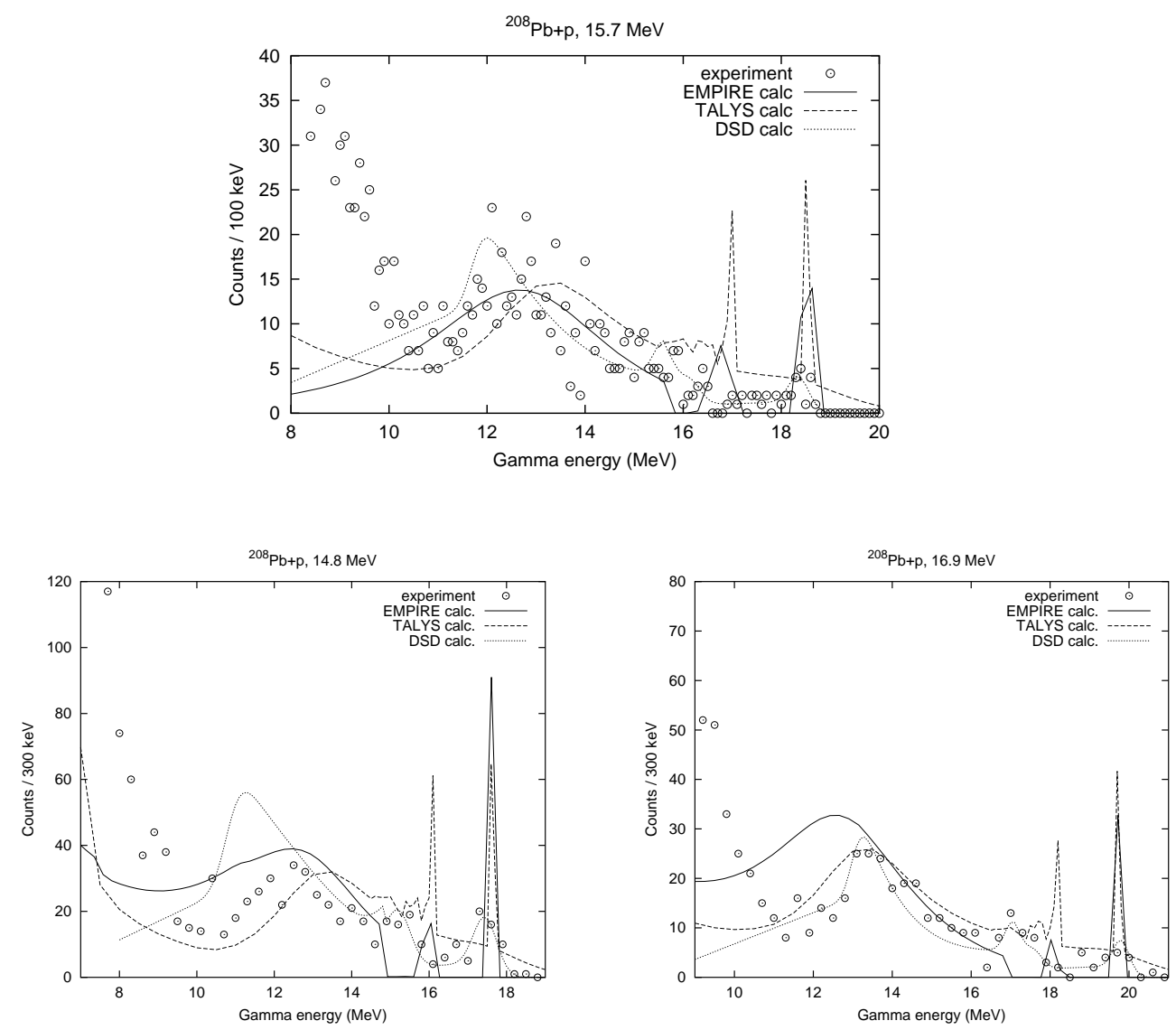

Figure 3: The $\gamma$ spectrum measured in coincidence with the $f_{7 / 2}(896 \mathrm{keV})$ transition in ${ }^{208} \mathrm{~Pb}+\mathrm{p}$ at 15.7 (top), 14.8 and 16.9 MeV (from Refs. [20, 30]).

\section{Conclusions}

We have pointed out some underlying features which lead to the appearance of the complex effective potential used in the optical model, and indicated its possible counterpart in the preequilibrium statistical model, in both cases applied to the nucleon radiative capture reactions at incident energies above $10 \mathrm{MeV}$. Both DSD and pre-equilibrium calculations have been compared to the data of the ${ }^{208} \mathrm{~Pb}(\mathrm{p}, \gamma)$ reactions leading to discrete states at incident energies ranging from few $\mathrm{MeV}$ to about $20 \mathrm{MeV}$. Therein, the statistical way of pre-equilibrium description does not work so nicely - it is explained by specific non-statistical behaviour in the vicinity of doublymagic ${ }^{208} \mathrm{~Pb}$. The two codes, EMPIRE and TALYS, used for the pre-equilibrium calculations give results of similar quality, and there is no clear dominance of one of them over the other.

\section{Acknowledgments}

This work has been supported in part by VEGA Grant No. 2/7115/27 and by the APVV project No. SK-SI-20-06. 


\section{References}

[1] G.E. Brown, Direct and semi-direct $(p, \gamma)$ and $(n, \gamma)$ reactions, Nucl. Phys. 57 (1964) 339.

[2] M. Potokar, The complex coupling interaction in the radiative capture of fast nucleons, Phys. Lett. 46B (1973) 346.

[3] A. Likar, T. Vidmar, Fast neutron capture through a consistent version of the direct-semidirect model, Nucl. Phys. A591 (1995) 458.

[4] E. Gadioli, P. Hodgson, Pre-Equilibrium Nuclear Reactions, Clarendon Press, Oxford 1992.

[5] E. Běták, J. Dobeš, Gamma emission in the pre-equilibrium exciton model, Phys. Lett. 84B (1979) 368.

[6] J.M. Akkermans, H. Gruppelaar, Analysis of continuum gamma-ray emission in precompound-decay reactions, Phys. Lett. 157B (1985) 95.

[7] F. Cvelbar, and E. Běták, Exciton model comparison of the activation and the integrated $14 \mathrm{MeV}$ neutron radiative capture cross sections, Z. Phys. A332 (1989) 163.

[8] F. Cvelbar, E. Běták, J. Merhar, Pre-equilibrium-equilibrium model calculations of nucleon radiative capture excitation functions, J. Phys. G17 (1991) 113.

[9] E. Běták, F. Cvelbar, J. Kopecky, Another possible manifestation of the energy-dependent width of giant dipole resonance, Phys. Rev. C46 (1992) 945.

[10] F. Cvelbar, E. Běták, A. Likar, Pre-equilibrium and direct-semi-direct model calculations of proton radiative capture excitation functions near A = 60, J. Phys. G19 (1993) 1937.

[11] F. Cvelbar, E. Běták, A. Likar, Pre-equilibrium and direct-semi-direct model calculations of nucleon radiative capture excitation functions on heavy nuclei, J. Phys. G21 (1995) 377.

[12] P. Obložinský, Preequilibrium $\gamma$ rays with angular momentum coupling, Phys. Rev. C35 (1987) 407.

[13] P. Obložinský, M.B. Chadwick, Gamma-ray emission from multistep compund reactions, Phys. Rev. C42 (1990) 1652.

[14] E. Běták, F. Cvelbar, A. Likar, T. Vidmar, Model calculations of the radiative capture process and the Brink-Axel hypothesis, Nucl. Phys. A686 (2001) 204.

[15] E. Běták, F. Cvelbar, A. Likar, and T. Vidmar, Radiative neutron and proton capture: Pre-equilibrium approach, in Proc. Eleventh Internat. Symp. Capture Gamma-Ray Spectroscopy and Related Topics, Pruhonice near Prague, 2-6 Sept. 2002", Eds. J. Kvasil, P. Cejnar, M. Krtička, World Scientific., Singapore, 2003, p. 212.

[16] M. Herman, P. Obložinský, R. Capote, A. Trkov, V. Zerkin, M. Sin, B. Carlson, EMPIRE modular system for nuclear reaction calculations (version 2.19 Lodi), Report NNDC-BNL, Upton, USA 2005.

[17] A. Koning, S. Hilaire, M. Duijvestijn, TALYS: A nuclear reaction program, Report 21297/04.62741/P FAI/AK/AK, NRG Petten 2004.

[18] E. Běták, PEQAG: A PC version of fully pre-equilibrium computer code with gamma emission, Report INDC(CSR)-016/LJ, IAEA Vienna 1989.

[19] E. Běták, P. Obložinský, PEGAS: Pre-equilibrium-equilibrium gamma-and-spin code (PC version), Report INDC(SLK)-001, IAEA Vienna 1993. 
[20] E. Běták, A. Likar, M. Lipoglavšek, M. Vencelj, T. Vidmar, in 11th Internat. Conf. Nucl. Reacts. Mechanisms, Varenna, June 12-16, 2006. Ed. E. Gadioli [Univ. Milano, Ric. Sci. Educ. Perm., Suppl. 126, Milano 2006], p. 203.

[21] Nuclear Data for the Production of Therapeutic Radionuclides. Summary Report of First Research Coordination Meeting. Prepared by J.-Ch. Sublet and R. Paviotti-Curcuera. Report INDC(NDS)-444, IAEA Vienna 2003.

[22] Nuclear Data for the Production of Therapeutic Radionuclides. Summary Report of Second Research Coordination Meeting. Prepared by J.-Ch. Sublet and R. Capote Noy. Report INDC(NDS)-465, IAEA Vienna 2004.

[23] K. Snover, J. Amann, W. Hering, P. Paul, Proton radiative capture into ${ }^{209}$ Bi through giant resonances and analog resonances, Phys. Lett. B37 (1971) 29.

[24] S.S. Hanna, in Proc. Int. Conf. Nucl. Phys. with Electromagntic Interacts., Mainz 1979. Eds. H. Arenhövel, D. Drechsel, Springer, Berlin, 1979, p. 288

[25] M. Lipoglavšek, R. Bark, M. Benatar, E. Gueorguieva, J. Kau, F. Komati, P. Kwinana, J. Lawrie, G. Mabala, P. Maine, S. Mukhrejee, S. Mullins, S. Murray, N. Ncapayi, R. Newman, P. Vymers, A. Likar, M. Vencelj, and T. Vidmar, High resolution measurement of the ${ }^{208} \mathrm{~Pb}(p, \gamma)$ capture reaction up to $E_{\gamma}=19 \mathrm{MeV}$, Phys. Lett. B593 (2004) 61.

[26] E. Běták: Model calculations of radiative capture of nucleons in MeV region, in Capture Gamma-Ray Spectroscopy and Related Topics: 12th International Symposium, Notre Dame, Indiana (USA), 4-9 Sept. 2005. Eds. A. Woehr, A. Aprahamian. AIP Conference Proc. vol. 819 (Amer. Inst. Phys., Melville, New York 2006).

[27] A. Likar, T. Vidmar, Efefctive interaction Hamiltonians in direct reactions, in NEMEA-3, 3rd Workshop on Neutron Measurements, Evaluations and Applications, Borovec, 25-28 October 2006 in NEMEA-3 (Neutron Measurements and Evaluations for Applications) Enlargement Workshop, 25-28 Oct. 2006, Borovets, Bulgaria. Ed. A.J.M. Plompen. EUR Report 22794 EN. IRMM JRC Geel, Belgium, 2007, p. 85.

[28] U. Fano, Effects of Configuration Interaction on Intensities and Phase Shifts, Phys. Rev. 124 (1961) 1866.

[29] A. Likar, M. Lipoglavšek, M. Vencelj, T. Vidmar, R. Bark, E. Gueorguieva, F. Komati, J. Lawrie, S. Maliage, S. Mullins, S. Murray, and T. Ramashidzha, Proton capture to continuum states of ${ }^{209}$ Bi, Phys. Rev. C73 (2006) 044609.

[30] E. Běták, A. Likar, T. Vidmar, Radiative nucleon capture - a dual approach, in Proc. Internat. Conf. Nuclear Data for Science and Technology, Nice, April 2007 (submitted). 